\title{
Comparing different thrombectomy techniques in five large-volume centers: a 'real world' observational study
}

\author{
Amélie Carolina Hesse, ${ }^{1}$ Daniel Behme, ${ }^{1}$ André Kemmling, ${ }^{2}$ Antonia Zapf, ${ }^{3}$ \\ Nils Große Hokamp, ${ }^{4}$ Isabelle Frischmuth, ${ }^{5}$ Ilko Maier, ${ }^{6}$ Jan Liman, ${ }^{6}$ Ioannis Tsogkas, ${ }^{1}$ \\ Jan-Hendrik Buhk, ${ }^{5}$ Julia Tran, ${ }^{1}$ Jens Fiehler, ${ }^{5}$ Anastasios Mpotsaris, ${ }^{4}$ Peter Schramm, ${ }^{2}$ \\ Ansgar Berlis, ${ }^{7}$ Michael Knauth, ${ }^{1}$ Marios-Nikos Psychogios ${ }^{1}$
}

\begin{abstract}
- Additional material is published online only. To view please visit the journal online (http://dx.doi.org/10.1136/ neurintsurg-2017-013394)
\end{abstract}

1 Institute for Diagnostic and Interventional Neuroradiology, University Medical Center Goettingen, Goettingen, Germany

${ }^{2}$ Institute of Neuroradiology, University Medical Center Schleswig-Holstein, Luebeck, Germany

${ }^{3}$ Department of Medical

Statistics, University Medical Center Goettingen, Goettingen, Germany

${ }^{4}$ Institute of Diagnostic and Interventional Radiology, University Hospital Cologne, Cologne, Germany

${ }^{5}$ Department of Diagnostic and Interventional Neuroradiology, University Medical Center, Hamburg-Eppendorf, Hamburg, Germany

${ }^{6}$ Department of Neurology, University Medical Center Goettingen, Goettingen,

Germany

${ }^{7}$ Department of Neuroradiology, Hospital Augsburg, Augsburg, Germany

\section{Correspondence to}

Dr Marios-Nikos Psychogios, Department of Neuroradiology, University Medical Center Goettingen, Goettingen, NS 37077, Germany; m. psychogios@med.unigoettingen.de

Received 8 August 2017 Revised 7 September 2017 Accepted 12 September 2017 Published Online First 28 September 2017

Check for updates

To cite: Hesse AC, Behme $D$, Kemmling A, et al. $J$ Neurolntervent Surg

2018:10:525-529.

\begin{abstract}
Background and purpose Thrombectomy has become the standard of care for acute ischaemic stroke due to large vessel occlusion. Aim of this study was to compare the radiological outcomes and time metrics of the various thrombectomy techniques.
\end{abstract}

Methods In this retrospective, multicenter study we analysed the data of 450 patients with occlusion of the anterior circulation, treated in five high-volume center from 2013 to 2016. The treatment techniques were divided in three categories: first-pass use of a large-bore aspiration-catheter; first-pass use of a stent-retriever; and primary combined approach (PCA) of an aspirationcatheter and stent-retriever. Primary endpoints were successful reperfusion and groin to reperfusion time. Secondary endpoints were the number of attempts and occurrence of emboli in new territory (ENT). The primary analysis was based on the intention to treat groups (ITT). Results The ITT-analysis showed significantly higher reperfusion rates, with $86 \%$ of successful reperfusion in the PCA-group compared with $73 \%$ in the aspiration group and $65 \%$ in the stent-retriever group. There was no significant difference in groin to reperfusion time regarding the used technique. The secondary analysis showed an impact of the technique on the number of attempts and the occurrence of ENTs. Lowest ENT rates and attempts were reported with the combined approach.

Conclusions The combined first-pass deployment of a stent-retriever and an aspiration-catheter was the most effective technique for reperfusion of anterior circulation large vessel occlusion. Our results correlate with the latest single-centrere studies, reporting very high reperfusion rates with $\mathrm{PCA}$ variations.

\section{INTRODUCTION}

Endovascular treatment of ischaemic stroke has become the standard of care in cases with underlying large vessel occlusion (LVO). ${ }^{12}$ One of the major reasons for the positive results of the so-called 'big five' studies was the high rate of cases treated with stent-retrievers. The overall rate of successful reperfusion was $71 \%$ in the five trials analysed by the Hermes collaborators. ${ }^{1}$ The importance of modern devices becomes even more evident when angiographic outcomes of the positive studies are compared with the so-called 'unhappy-triad' studies. ${ }^{3-5}$ The Interventional Management of Stroke III trial reported a rate of $44 \%$ for successful reperfusion in middle cerebral artery M1 occlusions. ${ }^{3}$ In the Local Versus Systemic Thrombolysis for Acute ischaemic Stroke trial, the rate of angiographically proven reperfusion was not even mentioned and in the Mechanical Retrieval and Recanalization of Stroke Clots Using Embolectomy study only 27\% of successful reperfusion was achieved. ${ }^{45}$ In all 'unhappy-triad' studies a stent-retriever was rarely used. ${ }^{6}$ As thrombectomy devices have evolved beyond stent-retrievers, the question of how to reach the best angiographic results remains unanswered. Several technical approaches have been published regarding mechanical thrombectomy and can be generally divided into three major categories: the intracranial use of a stent-retriever with simultaneous extracranial aspiration through a balloon guide catheter (BGC); the direct aspiration firstpass technique (ADAPT), where removal of the clot is achieved by intracranial aspiration through a large-bore aspiration-catheter without the use of a stent-retriever; ${ }^{7}$ and the primary combined approach (PCA) with simultaneous intracranial use of a stent-retriever and an aspiration-catheter and the option of additional extracranial aspiration through the guide catheter. ${ }^{8-10}$ Recent studies have compared the first two approaches, with inconclusive results regarding reperfusion outcomes. ${ }^{1112}$ Higher reperfusion rates have been reported for the combined approach in smaller retrospective trials. ${ }^{10}$ In an in-vitro comparison of ADAPT versus stent-retriever plus aspiration, the combined approach achieved significantly higher rates of favourable revascularisation. ${ }^{13}$

To analyse the efficacy of the various thrombectomy techniques, we compared the angiographic results from five high-volume German centers on an intention to treat (ITT) basis. An ITT analysis is crucial in the setting of thrombectomy, as successful reperfusion is often achieved by a combination of different rescue approaches. Thus, reporting and relating reperfusion rates that are specifically attributed to any of the techniques above require a first ITT analysis. 


\section{MATERIALS AND METHODS}

\section{Patient selection}

Patients were retrospectively selected from databases of two high-volume German centers from the time of the first use of a large-bore aspiration catheter in 2013 to 2016. All consecutive cases with an LVO were included from center A (Goettingen, 150 cases) and center B (Augsburg, 179 cases). Information from center A and centre B were extracted from prospective databases and re-evaluated regarding angiographic results by a core team, consisting of an experienced interventional neuroradiologist ( $>5$ years of experience, MP) and a medical student (AH). Neurological scores in center A were assessed by a certified stroke neurologist at hospital admission, hospital discharge and 90 days after stroke onset. Neurological scores in center B were assessed by a neurologist at hospital admission and hospital discharge. Three additional centers with expertise in stent-retrievers (C Hamburg, 33 cases), in the use of aspiration catheters (D Cologne, 41 cases) and the PCA (E Luebeck, 47 cases) contributed consecutive cases performed in 2015 and 2016. Patient information from the three additional centers were extracted from the institutional databases; angiographic outcomes were re-evaluated by local experienced interventional neuroradiologists; and neurological scores were assessed by a neurologist at hospital admission and hospital discharge. All five prospective databases were approved by the local institutional review boards. Patients' consent for treatment was obtained according to institutional guidelines. The board waived the need for a formal application or a separate consent concerning the inclusion in our retrospective study.

\section{Thrombectomy technique}

The first group (1. aspiration only) of our collective consisted of primary aspiration cases performed with the ADAPT technique. We used a NeuronMAX 088 (Penumbra, Alameda, CA, USA), an 8F Mach1 (Boston Scientific, Marlborough, MA, USA) or an $8 \mathrm{~F}$ Vista Brite Tip (Codman, Raynham, MA, USA) as guide catheters in this group, while an ACE (Penumbra) or SOFIA (Microvention, Tustin, CA, USA) were used as aspiration-catheters. Physicians either stuck to the ADAPT plan or switched to a rescue maneuver with the use of a stent-retriever after incomplete reperfusion with ADAPT. Rescue cases were assigned to a second group (2. aspiration + stent retriever rescue). The third group (3. stent-retriever only) consisted of patients treated with a stent-retriever, usually a Solitaire (Medtronic, Dublin, Ireland) and a BGC, mostly an 8F Cello (Medtronic). Again, if multiple attempts of this third technique were unsuccessful the physician could switch to a rescue maneuver with an aspiration-catheter. This group was named ' 4 . stent-retriever + aspiration rescue'. The fifth group of our study consisted of combined approach cases done with the primary, simultaneous use of a stent-retriever and an aspiration-catheter (5. PCA-group). An 8F Vista Brite Tip (Codman), a NeuronMAX 088 (Penumbra) or a Mach1 (Boston Scientific) were predominantly used as guide catheters in this group, while intracranial aspiration was frequently applied through an ACE (Penumbra), SOFIA (Microvention) or CATALYST (Stryker, Neurovascular, Mountain View, CA, USA) aspiration catheter. In most cases of this fifth group a Trevo ProVue (Stryker) stent-retriever was used for thrombectomy. The PCA-technique for this fifth group was not pre-specified: most cases in center A and center E were performed with the stent-retriever assisted vacuum-locked extraction (SAVE) technique, while in most cases in center B the so-called 'Solumbra' technique was used. ${ }^{1014}$

\section{Statistical analysis}

The aim of this retrospective, multicenter study is to compare different thrombectomy techniques. The various techniques were not prospectively assigned, it was up to the treating physician and center to decide which technique to use. The primary analysis is based on the ITT population with assignment to three groups: first-pass aspiration (groups 1 and 2); first-pass stent-retriever (groups 3 and 4); and PCA (group 5). Secondary analyses are performed on a per protocol (PP) basis consisting of the five groups: (aspiration only; aspiration + stent-retriever rescue; stent-retriever only; stent-retriever + aspiration rescue; and PCA. The primary hypothesis is that the PCA-group is more efficient than the other two thrombectomy methods regarding the two co-primary endpoints: superiority of reperfusion rates measured by the modified Thrombolysis in Cerebral Infarction (mTICI) score, with a score of $2 \mathrm{~b}$ and 3 representing successful reperfusion; and non-inferiority of times from groin puncture to successful reperfusion, with a non-inferiority threshold of 1200 $\mathrm{s}(20 \mathrm{~min})$. The non-inferiority threshold was chosen by the authors prior to analysis of the results. The PCA-group is more efficient if both co-primary null hypotheses are rejected. The most important secondary endpoints are the device passes, the frequency of emboli in new territories (ENT), of symptomatic intracranial haemorrhages $(\mathrm{sICH})$ and the difference of National Institutes of Health Strokes Scale (NIHSS) and modified Rankin scale (mRS) from hospital admission to discharge.

Continuous study parameters are checked for normality using grouped boxplots for visualisation. If a normal distribution can be assumed, the groups are compared with a single factor analysis of variance (ANOVA), otherwise with a Kruskal-Wallis test. Categorical variables are compared with the chi-square or Fisher's exact test. All pvalues of baseline comparisons are used as descriptive measures.

The primary analyses consist of a logistic regression analysis of the co-primary endpoint 'successful reperfusion'. For the log-transformed co-primary endpoint 'groin to reperfusion time' a linear regression analysis is used. Because the two endpoints are co-primary, an adjustment of the type-one error is not needed. The different thrombectomy methods are included as independent variables while the baseline variables, which tend to be different in the groups $(\mathrm{p}<0.2)$, are included as covariates. For the pairwise comparisons of the thrombectomy methods a multiplicity adjustment is not needed because of the closed testing procedure. To assess the non-inferiority for the endpoint 'groin to reperfusion time' the pairwise location shifts with corresponding confidence intervals (CI) is calculated using the Hodges-Lehmann estimation.

Secondary endpoints are evaluated with descriptive statistics and regression analyses. While dichotomous endpoints (ENT, sICH) are analysed the same way as the endpoint 'successful reperfusion', we analyse metric endpoints (NIHSS, mRS) the same way as the endpoint 'groin to reperfusion time'. In further secondary analyses all aforementioned analyses are performed using the data of the PP-population.

\section{RESULTS}

Four hundred and fifty patients were included in our study (235 male, 52\%). Baseline characteristics are depicted in table 1 . The frequencies of the different techniques were relatively balanced. Of the 450 included patients, 164 (36\%) were assigned to the aspiration group, $102(23 \%)$ to the stent-retriever group and $184(41 \%)$ to the PCA-group. Regarding the comparison of ITTand PP-analysis it is noteworthy that while $10 \%$ of the stent-retriever group required a rescue maneuver, $34 \%$ of the aspiration 
Table 1 Baseline characteristics of patients between treatment groups (ITT population)

\begin{tabular}{|c|c|c|c|c|c|}
\hline Variable* & Overall $(n=450)$ & Aspiration $(n=164)$ & Stent-retriever $(n=102)$ & PCA $(n=184)$ & $p$ value \\
\hline Age (median, IQR) & $74,62-80$ & $72,60-79$ & $74,67-80$ & $75,65-81$ & $0.09+$ \\
\hline Male sex & $235(52)$ & $75(46)$ & $57(56)$ & $103(56)$ & $0.11 \neq$ \\
\hline Hypertension & $341(77)$ & $115(71)$ & $81(80)$ & $145(80)$ & $0.07 \ddagger$ \\
\hline Dyslipidemia & $169(38)$ & $62(38)$ & $37(37)$ & $70(39)$ & $0.93 \ddagger$ \\
\hline Diabetes mellitus & $106(24)$ & $33(20)$ & $26(26)$ & $47(26)$ & $0.39 \ddagger$ \\
\hline Atrial fibrillation & $199(45)$ & $75(46)$ & $44(44)$ & $80(45)$ & $0.92 \ddagger$ \\
\hline Peripheral artery occlusive disease & $23(6)$ & $10(6)$ & $7(7)$ & $6(4)$ & $0.65 \ddagger$ \\
\hline $\begin{array}{l}\text { NIHSS on admission } \\
\text { (mean, SD) }\end{array}$ & $15.1 \pm 5.8$ & $15.3 \pm 6.1$ & $15.5 \pm 5.5$ & $14.6 \pm 5.8$ & $0.40 \S$ \\
\hline $\begin{array}{l}\text { CCT-ASPECT } \\
\text { (median, IQR) }\end{array}$ & $8,7-9$ & $8,7-9$ & $8,7-9$ & $8,7-9$ & $0,29+$ \\
\hline Occlusion of intracranial carotid artery & $145(33)$ & $54(33)$ & $27(26)$ & $64(35)$ & $0.06 \ddagger$ \\
\hline Rescue maneuver & $66(15)$ & $56(34)$ & $10(10)$ & - & - \\
\hline
\end{tabular}

${ }^{*}$ Data are presented as number (\%) unless otherwise specified.

tpvalue of the Kruskal -Wallis test.

łpvalue of the chi square test.

§pvalue of the ANOVA.

cases needed an additional stent-retriever rescue (table 1). Vessel occlusion location was balanced with an internal carotid artery (ICA), an M1 and an M2 occlusion in 33\%, 60\% and 7\% of the aspiration group; in $26 \%, 65 \%$ and $9 \%$ of the stent-retriever group; and in 34\%, 53\% and $12 \%$ of the PCA-group. Successful reperfusion was documented in 119 (73\%) patients of the aspiration group, in $66(65 \%)$ patients of the stent-retriever group and in 159 (86\%) patients of the PCA-group. The results of the logistic regressions are shown in table 2 . The co-variables age, sex and hypertension have no significant effect on the reperfusion rate. Thrombectomy technique has a significant effect on reperfusion with an OR of 2.53 (95\% CI 1.45 to $4.43 ; \mathrm{p}=0.002$ ) for PCA compared with the aspiration group and an OR of 3.48 (95\% CI 1.92 to 6.32 ; $\mathrm{p}<0.001)$ for PCA compared with the stent-retriever group. A median groin to reperfusion time of $3240 \mathrm{~s}(54 \mathrm{~min})$ (IQR 32-81), of $3600 \mathrm{~s}(60 \mathrm{~min})$ (45-75) and of $3060 \mathrm{~s}(51 \mathrm{~min})(36-83)$ was documented in the aspiration group, in the stent-retriever group and in the PCA-group, respectively. The calculated CI for the location-shift were all between -20 and 20 (Online table I), thus non-inferiority is proven. The results of the linear regression show a relevant, but not significant, effect of the technique on time from groin to reperfusion and a relevant effect of age (Online table II). Taking the confounding factors (age, sex and hypertension) into account the median groin to reperfusion time for the aspiration group was $2940 \mathrm{~s}$ (49 min), for the stent-retriever group $3720 \mathrm{~s}$

Table 2 Results of the primary multiple logistic regression analysis for the 'successful reperfusion' in the ITT population

\begin{tabular}{lllll}
\hline & Comparison & OR & 95\% Cl (OR) & pvalue \\
\hline Technique & Global & - & - & $<0.01$ \\
& PCA versus aspiration & 2.53 & 1.45 to 4.43 & $<0.01$ \\
& PCA versus stent-retriever & 3.48 & 1.92 to 6.32 & $<0.01$ \\
& Aspiration versus stent- & 1.38 & 0.80 to 2.36 & 0.19 \\
Age & retriever & & & \\
Sex & Per year & 1.01 & 0.99 to 1.03 & 0.48 \\
Hypertension & Male versus female & 1.07 & 0.68 to 1.71 & 0.76 \\
\hline
\end{tabular}

(62 min) and for the PCA-group $3300 \mathrm{~s}$ (55 min). Regarding the ratios of the geometric means, the stent-retriever technique takes the longest (1.26 times longer than the aspiration and 1.13 times longer than the PCA), while the PCA takes 1.11 times longer than the aspiration technique (Online table III).

Descriptive parameters of the secondary endpoints are shown in table 3. The various techniques show no effect on the frequency of sICH, the delta of admission and discharge NIHSS or mRS values. Less device-passes and ENT were documented with the PCA technique. Regarding mTICI3 frequencies, the PCA-approach showed the highest percentage with $37.5 \%$ (69/184), followed by the aspiration group with $27.5 \%(45 / 164)$ and the stent-retriever group with 19\% (19/102). First-pass mTICI3 was $26 \%$ (47/184), 17\% (28/161) and 14\% (14/102) for the PCA-, aspiration and stent-retriever group, respectively.

The baseline characteristics of the PP-analysis are shown on table 4. Relevant differences are documented for age and hypertension but not for sex. Regarding the various thrombectomy techniques, aspiration only and PCA had high reperfusion rates with $81 \%$ and $86 \%$, while aspiration with stent-retriever rescue show the lowest rates with 57\% (Online table IV). The OR were calculated pairwise against the PCA-group and can be seen in Online table V. The aspiration only group showed the shortest groin to reperfusion time with $2520 \mathrm{~s}$ (42 min) (IQR 27-65) followed by the PCA-group with $3060 \mathrm{~s}$ (51 min) (IQR 36-83). The longest groin to reperfusion times were documented in the aspiration plus stent-retriever rescue group with $4980 \mathrm{~s}$ (83 min) (IQR 63-135).

\section{DISCUSSION}

In this series, we report the reperfusion results and interventional time metrics of 450 patients from five large-volume German centers since the first use of a large-bore aspiration catheter in 2013. We retrospectively observed consecutive thrombectomies in a 'real world' scenario, performed by interventionalists with variable experience (from first-year senior to chief of interventional therapies). Thrombectomy techniques were divided into three main categories (aspiration, stent-retriever and PCA) and analysed on an ITT basis. As both co-primary null-hypotheses are rejected, our results support the notion that the PCA approach is the best thrombectomy technique in the treatment of LVO. 
Table 3 Descriptive statistics of the secondary endpoints in the overall group and in the individual groups (ITT population)

\begin{tabular}{|c|c|c|c|c|c|}
\hline Endpoint* & Overall & Aspiration & Stent-retriever & PCA & pvalue \\
\hline $\mathrm{sICH}$ & $29(7.3)$ & $11(7.0)$ & $5(5.1)$ & $13(9.2)$ & 0.42 \\
\hline ENT & $41(9.2)$ & $19(11.6)$ & $10(9.8)$ & $12(6.6)$ & 0.19 \\
\hline $\begin{array}{l}\text { Number of device passes } \\
\text { (median, IQR) }\end{array}$ & $2,1-3$ & $2,1-4$ & $2,1-4$ & $2,1-3$ & $\begin{array}{l}<0.01 \\
\text { Asp. versus stent-ret.: } 0.58 \\
\text { Asp. versus PCA: }<0.01 \\
\text { Stent-ret. versus PCA: } 0.01\end{array}$ \\
\hline Delta NIHSS (mean, SD) † & $-6.9 \pm 6.8$ & $-7.1 \pm 6.8$ & $-6.1 \pm 7.4$ & $-7.2 \pm 6.3$ & 0.42 \\
\hline Delta mRS (mean, SD) $\dagger$ & $-1.1 \pm 1.7$ & $-1.2 \pm 1.8$ & $-0.8 \pm 1.8$ & $-1.1 \pm 1.7$ & 0.39 \\
\hline
\end{tabular}

*Data are presented as number (\%) unless otherwise specified.

tchange from admission to discharge. p-values are from the multiple regression analysis with age, sex and hypertension as covariates.

Combined mTICI $2 \mathrm{~b}$ and 3 (86\%), mTICI 3 (37.5\%) and firstpass mTICI 3 (26\%) were all higher for the PCA-group. Simultaneously, the PCA approach did not show significantly longer groin to reperfusion times since the interventionalists needed 1.11 times longer for a PCA-thrombectomy compared with the aspiration technique. The longest times were reported for the stent-retriever group with 1.26 times longer periods, compared with the aspiration metrics, and 1.13 times longer interventions, compared with the PCA-group.

Thrombectomy combined with thrombolysis, if eligible, has become the new standard therapy of $\mathrm{LVO}$ after publication of the multiple positive trials in 2015. ${ }^{15-19}$ Although available evidence shows superiority of stent-retrievers versus older devices such as the MERCI device, there are not many comparisons of stent-retrievers versus new-generation recanalisation devices such as large-bore aspiration-catheters or the combination of both. ${ }^{2021}$ A recently published randomised trial showed non-inferiority of direct aspiration with a large-bore aspiration-catheter compared with the use of a stent-retriever. ${ }^{12}$ However, multiple smaller observational studies published in the past few years showed very high reperfusion rates $(84 \%-100 \%)$ for the combination of both devices. ${ }^{8-10} 14$ Accordingly, more and more interventionalists use the PCA, which results in increased costs due to the simultaneous use of two devices. ${ }^{22}$ Our study results support the combined use of two thrombectomy devices as the primary approach. Interestingly, the results of the PCA-group in our series are worse than those published in recent single-center studies. ${ }^{8-10}$ A possible explanation is the 'real world' design of our series, containing thrombectomies performed by interventionalists of various experience grades, as opposed to a more controlled/selected design of smaller studies, performed only by very experienced neurointerventionalists. The 'real world' design also reflects the fact that various PCAs were used in our study ('Solumbra', SAVE etc.), in contrast to a standardised approach of smaller studies describing a new technique. While the results of our aspiration group are comparable to the results of the Randomized, Concurrent Controlled Trial to Assess the Penumbra System's Safety and Effectiveness in the Treatment of Acute Stroke trial (70\%), they are worse than the results of the recently published ASTER trial $(85.4 \%) .{ }^{12}{ }^{23}$ Likely explanations for this discrepancy could be the 'real world' design of our series as well as the frequencies' discrepancy of ICA occlusions treated in our study (with 30\%) as opposed to the frequency in the ASTER trial (with 10\%). Clot length and overall mass is bigger in ICA occlusions leading to more device passes and lower reperfusion rates. ${ }^{24}$ The results of our stent-retriever group correlate well with the angiographic results published in the MR-CLEAN and THRACE trials. ${ }^{15} 25$ The stent-retriever group results are worse than the EXTEND-IA or SWIFT PRIME reperfusion rates. However, the frequency of ICA occlusion in the SWIFT PRIME trial was much lower than in our series ( $\sim 30 \%$ vs $\sim 15 \%)$.

Although device passes were significantly lower with a PCA in our series, we did not observe any difference in the NIHSS or mRS deltas from admission to discharge. ${ }^{26}$ Unfortunately, 90 day mRS was not available for $\sim 50 \%$ of our patients, which means that a possible clinical effect of better reperfusion results in later stages of rehabilitation was not documented in our series. We did, however, observe a tendency towards lower ENT in the PCA-group.

Table 4 Baseline characteristics secondary analysis of per protocol population

\begin{tabular}{|c|c|c|c|c|c|c|}
\hline Variable* & Technique & & & & & \\
\hline & $1(n=108)$ & $2(n=56)$ & $3(n=92)$ & $4(n=10)$ & $5(n=184)$ & $p$ value \\
\hline Age (median, IQR) & $74,61-80$ & $71,59-78$ & $74,66-80$ & $77,76-80$ & $75,65-81$ & 0.13 \\
\hline NIHSS admission (mean, SD) & $15.5 \pm 6.4$ & $14.9 \pm 5.4$ & $15.5 \pm 5.6$ & $15.1 \pm 4.8$ & $14.6 \pm 5.8$ & $0.67 \dagger$ \\
\hline Male & $50(46)$ & $25(45)$ & $53(58)$ & $4(40)$ & $103(56)$ & $0.24 \ddagger$ \\
\hline Hypertension & $73(68)$ & $42(76)$ & $72(78)$ & $9(100)$ & $145(80)$ & $0.06 \ddagger$ \\
\hline Dyslipidaemia & $39(36)$ & $23(42)$ & $32(35)$ & $5(56)$ & $70(39)$ & $0.71 \neq$ \\
\hline Diabetes mellitus & $22(20)$ & $10(20)$ & $25(27)$ & $1(11)$ & $47(26)$ & $0.55 \ddagger$ \\
\hline Artrial fibrilliation & $50(46)$ & $25(45)$ & $41(45)$ & $3(33)$ & $80(45)$ & $0.96 \ddagger$ \\
\hline Peripheral artery occlusive disease & $5(5)$ & $5(9)$ & $7(8)$ & $0(0)$ & $6(4)$ & $0.55 \ddagger$ \\
\hline
\end{tabular}

Technique $1=$ aspiration, 2 =aspiration + stent-retriever rescue, $3=$ stent retriever, $4=$ stent retriever+aspiration rescue, $5=P C A$.

${ }^{*}$ Data are presented as number (\%) unless otherwise specified.

$t=p$-value of the ANOVA.

$\ddagger=p$-value of the chi square test. 
Limitations of our study include the retrospective, observational design and the non-standardised fashion of thrombectomy. Despite categorising all interventions in three common groups, we cannot exclude effects on reperfusion rates through small details (such as additional extracranial aspiration, distal stent-retriever placement etc.). Although $73 \%$ of the interventions were analysed by a core-laboratory, there was a part of angiographic outcomes being self-reported by the individual centres. The lack of $90 \mathrm{~d} \mathrm{mRS}$ prohibits a complete evaluation of the clinical impact of our angiographic and workflow findings.

\section{SUMMARY/CONCLUSIONS}

Thrombectomy technique has a significant effect on reperfusion rates without significantly affecting groin to reperfusion times. Our series supports the use of both a stent-retriever and an aspiration catheter as a first-pass strategy in LVO therapy.

Contributors Conception and design: ACH, M-NP. Analysis and interpretation of data: ACH, M-NP, DB, AK, AZ, NGH, IF, IM, JL, IT. Drafting of the manuscript: ACH, M-NP, J-HB, JT, JF, AM, PS, AB. Statistics: ACH, M-NP, AZ. Critically revising and approval of the manuscript: all authors.

Competing interests Jens Fiehler has received fees as consultant or lecturer from Acandis, Bayer, Boehringer-Ingelheim, Codman, Covidien, Medtronic, MicroVention, Penumbra, Philips, Sequent, Siemens and Stryker. Lectures for Boehringer-Ingelheim, Covidien, and Penumbra. Funding to institution: Medtronic, MicroVention. Funding to Institution: DFG, EU, BMBF,BMWi. Jan-Hendrik Buhk has received fees as consultant orlecturer from Acandis, Bayer, Codman, Medtronic, MicroVention, Philips and Stryker.Michael Knauth has received fees as consultant or lecturer from Siemens, Penumbra, Stryker, Acandis, Bayer.Marios-Nikos Psychogios has received fees as consultant or lecturer from Siemens, Penumbra, Stryker.

Ethics approval Ethics committee University Medical Centre Goettingen.

Provenance and peer review Not commissioned; externally peer reviewed.

Data sharing statement Yes, we are willing to provide additional data upon request.

(c) Article author(s) (or their employer(s) unless otherwise stated in the text of the article) 2018. All rights reserved. No commercial use is permitted unless otherwise expressly granted.

\section{REFERENCES}

1 Goyal M, Menon BK, van Zwam WH, et al. Endovascular thrombectomy after large-vessel ischaemic stroke: a meta-analysis of individual patient data from five randomised trials. Lancet 2016;387:1723-31.

2 Fiehler J, Cognard C, Gallitelli M, et al. European Recommendations on Organisation of Interventional Care in Acute Stroke (EROICAS). Int J Stroke 2016;11:701-16.

3 Broderick JP, Palesch YY, Demchuk AM, et al. Endovascular therapy after intravenous t-PA versus t-PA alone for stroke. N Engl J Med 2013;368:893-903.

4 Kidwell CS, Jahan R, Gornbein J, et al. A trial of imaging selection and endovascular treatment for ischemic stroke. N Engl J Med 2013;368:914-23.

5 Ciccone A, Valvassori L, Nichelatti M, et al. Endovascular treatment for acute ischemic stroke. N Engl J Med 2013;368:904-13.

6 Ding D. Endovascular mechanical thrombectomy for acute ischemic stroke: a new standard of care. J Stroke 2015;17:123-6.
7 Turk AS, Spiotta A, Frei D, et al. Initial clinical experience with the ADAPT technique: a direct aspiration first pass technique for stroke thrombectomy. J Neurointerv Surg 2014;6:231-7.

8 Massari F, Henninger N, Lozano JD, et al. ARTS (Aspiration-Retriever Technique for Stroke): initial clinical experience. Interv Neuroradiol 2016;22:325-32.

9 McTaggart RA, Tung EL, Yaghi S, et al. Continuous aspiration prior to intracranial vascular embolectomy (CAPTIVE): a technique which improves outcomes. J Neurointerv Surg 2016:neurintsurg-2016-012838.

10 Maus V, Behme D, Kabbasch C, et al. Maximizing first-pass complete reperfusion with SAVE. Clin Neuroradiol 2017.

11 Frei D, Nogueira R, Kirmani J, et al. Per protocol versus intent-to-treat analysis in a non-inferiority, randomized trial of the safety and efficacy of the penumbra 3D stent retriever as an adjunct to aspiration in acute ischemic stroke. Stroke 2017;48(Suppl 1):A32.

12 Lapergue B, Blanc R, Gory B, et al. Effect of endovascular contact aspiration vs stent retriever on revascularization in patients with acute ischemic stroke and large vessel occlusion: the ASTER randomized clinical trial. JAMA 2017;318:443-52.

13 Mokin M, lonita CN, Nagesh SV, et al. Primary stentriever versus combined stentriever plus aspiration thrombectomy approaches: in vitro stroke model comparison. J Neurointerv Surg 2015;7:453-7.

14 Delgado Almandoz JE, Kayan Y, Young ML, et al. Comparison of clinical outcomes in patients with acute ischemic strokes treated with mechanical thrombectomy using either Solumbra or ADAPT techniques. J Neurointerv Surg 2016;8:1123-8.

15 Berkhemer OA, Fransen PS, Beumer D, et al. A randomized trial of intraarterial treatment for acute ischemic stroke. N Engl J Med 2015;372:11-20.

16 Goyal M, Demchuk AM, Menon BK, et al. Randomized assessment of rapid endovascular treatment of ischemic stroke. N Engl J Med 2015;372:1019-30.

17 Saver JL, Goyal M, Bonafe A, et al. Stent-retriever thrombectomy after intravenous t-PA vs. t-PA alone in stroke. N Eng/ J Med 2015;372:2285-95.

18 Campbell BC, Mitchell PJ, Kleinig TJ, et al. Endovascular therapy for ischemic stroke with perfusion-imaging selection. N Engl J Med 2015;372:1009-18.

19 Molina CA, Chamorro A, Rovira À, et al. REVASCAT: a randomized trial of revascularization with SOLITAIRE FR device vs. best medical therapy in the treatment of acute stroke due to anterior circulation large vessel occlusion presenting within eight-hours of symptom onset. Int J Stroke 2015;10:619-26.

20 Powers WJ, Derdeyn CP, Biller J, et al. 2015 American Heart Association/American Stroke Association focused update of the 2013 guidelines for the early management of patients with acute ischemic stroke regarding endovascular treatment: a guideline for healthcare professionals from the American Heart Association/American Stroke Association. Stroke 2015 46:3020-35.

21 Psychogios MN, Kreusch A, Wasser K, et al. Recanalization of large intracranial vessels using the penumbra system: a single-center experience. AJNR Am J Neuroradiol 2012;33:1488-93.

22 Shireman TI, Wang K, Saver JL, et al. Cost-effectiveness of solitaire stent retriever thrombectomy for acute ischemic stroke: results from the SWIFT-PRIME trial (Solitaire With the Intention for Thrombectomy as Primary Endovascular Treatment for Acute Ischemic Stroke). Stroke 2017;48:379-87.

23 Mocco J, Zaidat 00, von Kummer R, et al. Aspiration thrombectomy after intravenous alteplase versus intravenous alteplase alone. Stroke 2016;47:2331-8.

24 Blanc R, Redjem H, Ciccio G, et al. Predictors of the Aspiration Component Success of a Direct Aspiration First Pass Technique (ADAPT) for the endovascular treatment of stroke reperfusion strategy in anterior circulation acute stroke. Stroke 2017:48:1588-93.

25 Bracard S, Ducrocq X, Mas JL, et al. Mechanical thrombectomy after intravenous alteplase versus alteplase alone after stroke (THRACE): a randomised controlled trial. Lancet Neurol 2016;15:1138-47.

26 Seker F, Pfaff J, Wolf M, et al. Correlation of thrombectomy maneuver count with recanalization success and clinical outcome in patients with ischemic stroke. AJNR Am J Neuroradio/ 2017;38:1368-71. 